NASA Contractor Report 185143

\title{
Mechanics of the Crack Path Formation
}

\author{
Asher A. Rubinstein \\ Tulane University \\ New Orleans, Louisiana
}

September 1989

Prepared for

Lewis Research Center

Under Grant NAG3-815

\section{N/Sก \\ National Aeronautics and \\ Space Administration}

$$
\begin{aligned}
& \text { (NASA-CR-185143) MECHANICS UF THF CRACK } \\
& \text { PATH FORMATION Finul ReDort (Tulane Univ.) } \\
& 21 \mathrm{D} \\
& \text { CSCL PUK }
\end{aligned}
$$





\title{
MECHANICS OF THE CRACK PATH FORMATION
}

\author{
Asher A. Rubinstein \\ Department of Mechanical Engineering \\ Tulane University \\ New Orleans, LA 70118
}

\begin{abstract}
A detailed analysis of experimentally obtained curvilinear crack path trajectories formed in a heterogeneous stress field is presented. The experimental crack path trajectories, described in [1], were used as data for the numerical simulations, recreating the actual stress field governing the development of the crack path. Thus, the current theories of crack curving and kinking could be examined by comparing them with the actual stress field parameters as they develop along the experimentally observed crack path. The experimental curvilinear crack path trajectories were formed in the tensile specimens with a hole positioned in the vicinity of a potential crack path [1]. The numerical simulation, based on the solution of equivalent boundary value problems with the possible perturbations of the crack path, is presented here.
\end{abstract}

\section{Introduction}

A commonly observed curvilinear crack propagation pattern is attributed to a mixed mode stress state in the vicinity of the crack tip. Generally, a curvilinear, wave-like, crack path is beneficial for material toughening [2]. The understanding of the mechanics of crack path kinking and curving is important for future design of material compositions capable of deflecting the crack propagation trajectory. This work aims to evaluate the crack path deflection criteria described in the literature and to state typical aspects of the experimentally observed crack path trajectory formation features. The approach undertaken here is essentially different from existing work of similar aims. This investigation is based strictly on experimental observations which are interpreted through a numerical analysis of the observed crack patterns with finite length of the curving segments. This makes it different from crack path analysis or predictions based on an infinitesimally 
small segment of crack kinking and curving, as compared with the length scale of the problem [3-16].

The experimental data were obtained and reported by Chudnovsky et al. [1]. Briefly, the experimental procedure can be described as follows:

A polystyrene single notch specimen with a hole positioned near the expected crack path (Fig. 1a) was subjected to a low cycle fatigue loading. Cycle loading was used to ensure stable crack growth. This particular material does not exhibit a time dependent fracture mechanism which could be attributed to fatigue crack propagation; therefore, in the described experiment, cycle loading was used to prevent dynamic unstable crack growth. Several geometries were used to obtain different intensities of crack hole interaction and to ensure that the region of this interaction would be confined to the center of the specimen, thus preventing influence from the ends of the specimens. The crack propagation described in [1] is accompanied by a damage zone typical for this material, the mechanical nature of which is formation of crazes. The typical size of the damage zone is significantly smaller than the geometrical parameter of the problem (diameter of the hole). Thus, two observations are possible; observation on microscale and on macroscale. The first, which was a primary subject of [1], is aimed at processes typical to the region in the vicinity of the propagating crack tip, so the size of the damage zone becomes the length parameter of the observed physical process. The latter considers the crack with the adjacent propagating damage zone as one crack interacting with a hole. This would be the view of an observer from a certain distance, when the damage zone appears just as some fuzziness along the crack surface. Naturally, the results of the analysis on macroscale can serve as boundary value data for the analysis on microscale.

A summary of the experimental data, observed in [1], is given in Figure 2. Six experimental curves, normalized by the hole radius, in the effective region are given. Three different cases of the crack interaction with a hole are represented here: crack attraction, cases one, six and five; attraction and repulsion, cases three and two; and, finally, no effect 
on the crack path, case four (the numbering sequence here has no significance; it is left in the given form for data reference only).

\section{Analytical Formulation and Numerical Procedure.}

The dimension of the area of crack - hole interaction is significantly smaller than the crack length, and smaller than the width of the specimen, as well. The size of this area can be judged by the distance from the hole to the first crack path deviation from the rectilinear trajectory. Therefore, the effective region can be assumed to be located in the infinite plate and to be governed by the applied stress intensity factor. Thus, the small scale model may be employed. The applied (or remote) stress intensity factor in this case is the stress intensity factor which would act on a straight crack in an equal plate without a hole. The length of this crack is equal to a projection of the curvilinear crack onto a horizontal direction, Figure 1, a) and b). Thus, the solutions for the local (actual) stress intensity factors and other crack growth criteria are obtained and given as in nondimensional form, in most cases as a value normalized by the applied stress intensity factor $K_{\mathrm{I}}{ }^{a}$.

The stress field of a curvilinear crack interacting with a hole is obtained by employing the numerical approach developed for a small scale model in $[2,18]$. The stress field is represented by analytic potentials $\phi(z)$ and $\psi(z)$, Muskhelishvili [17]. The stress tensor components in the coordinate system tangential $(\eta, \xi)$ to the crack surface can be written as

$$
\begin{aligned}
& \sigma_{\eta \eta}+i \sigma_{\xi \eta}=\phi^{\prime}+\bar{\phi}^{\prime}+\mathrm{e}^{2 i \theta}\left(\bar{z}_{\phi^{\prime}}(z)+\psi^{\prime}(z)\right), \\
& \sigma_{\xi \xi}-i \sigma_{\xi \eta}=\phi^{\prime}+\bar{\phi}^{\prime}-\mathrm{e}^{2 i \theta}\left(\bar{z} \phi^{\prime \prime}(z)+\psi^{\prime}(z)\right) .
\end{aligned}
$$

where $\theta$ is the angle between the positive direction of $x$ axis and tangential to the crack surface $\xi$.

The crack is represented as an array of dislocations along its curvilinear trajectory. Resulting stress potentials are 


$$
\begin{aligned}
& \phi^{\prime}(z)=\frac{\alpha}{2 \pi i} \int_{L}\left[\frac{b(s)}{z-t}+\phi_{s}^{\prime}(b(s), z, t)\right] d s, t=t_{x}(s)+i t_{y}(s) \\
& \psi^{\prime}(z)=-\frac{\alpha}{2 \pi i} \int_{L}\left[\frac{\overline{b(s)}}{z-t}-\frac{\overline{t b}(s)}{(z-t)^{2}}-\psi_{s}^{\prime}(b(s), z, t)\right] d s
\end{aligned}
$$

Here, the integrals are taken along the crack surface $L$ with $s$, a line length of the crack path starting at the crack tip, being chosen as an integration variable. $b(s)$ is an unknown dislocation density distribution along the crack, and $t$ is an integration point on the crack corresponding to the integration variable $s . \alpha$ here is a standard coefficient, $\alpha=E / 4\left(1-\nu^{2}\right)$ in plain strain case, and $\alpha=E / 4$ in plain stress case. The singular terms under the integrals (2.3) and (2.4) represent singular parts of stress potentials corresponding to a dislocation with Burgers vector $b(s) \mathrm{d} s$ positioned at $t$. The analytic functions $\phi_{\mathrm{S}}$ and $\psi_{\mathrm{S}}$ represent regular parts of these potentials resulting from the interaction of the dislocation with a hole, thus satisfying the traction free boundary conditions on the hole surface. These potentials are, [18],

$$
\begin{aligned}
& \phi_{S}^{\prime}(b, z, t,)=\hat{\phi}_{S}^{\prime}(b, z-a, t-a) \\
& \psi_{S}^{\prime}(b, z, t)=\hat{\psi}_{S}^{\prime}(b, z-a, t-a)-\bar{a}_{\dot{S}}^{*}(b, z-a, t-a)
\end{aligned}
$$

where $a$ is center of the hole, $R$ is the radius of the hole, and potentials corresponding to the case $a=0$ are

$$
\begin{aligned}
& \hat{\phi}_{s}^{\prime}(b, z, t)=\frac{\alpha}{2 \pi i}\left[b\left(\frac{1}{z}-\frac{1}{z-t^{*}}\right]+\frac{\bar{b}\left(t^{*}-t\right) t^{*}}{\bar{t}\left(z-t^{*}\right)^{2}}\right] \\
& \hat{\psi}_{s}^{\prime}(b, z, t)=-\frac{\alpha}{2 \pi i}\left[b\left[\frac{1}{z}-\frac{1}{z-t^{*}}\right]+\frac{\bar{b} R^{2}}{t z^{2}}\right]-\frac{R^{2}}{z} \phi_{s}^{*}+\frac{R^{2}}{z^{2}} \phi_{s}^{\prime} \\
& t^{*}=R^{2} / \bar{t}
\end{aligned}
$$

here $t$ is a current location of the dislocation.

The statement of a traction free crack surface,

$$
\sigma_{\eta \eta}+i \sigma_{\xi \eta}=0,
$$

leads to the integral equation for determination of the unknown dislocation distribution 
density function $b(s)$. The integral equation is obtained by substitutions of (2.3) and (2.4), with the use of (2.5-8), into (2.1) and then satisfying (2.9). The resulting integral equation is too long to reproduce in explicit form, and not all the details are important for development of the numerical procedure. The resulting equation can be written in the form

$$
f_{0}^{\infty}\left[\frac{N(b(s), \overline{b(s)})}{t(s)-z(v)}+P(\overline{b(s)}, b(s), K(s, v))\right] d s=0 .
$$

Functions $N$ and $P$ in (2.10) are linear functions of their arguments. $K(s, v)$ here is a Fredholm type kernel of the integral equation (2.10). In reality, functions $b(s)$ and the conjugate to it have different Fredholm kernels not shown in (2.10) explicitly in order to emphasize just the essential features of the equation. Important components of it are functions $t(s)$ and $z(v)$. These functions represent the transformation of the integral equation in a complex plane into a line integral equation in terms of real variables $s$ and $v$ along the crack path $L$. An important restriction on possible crack trajectories is a requirement that

$$
|\mathrm{t}(\mathrm{s})-\mathrm{z}(\mathrm{v})| \rightarrow \text { const }|\mathrm{s}-\mathrm{v}| \text { as }(\mathrm{s}-\mathrm{v}) \rightarrow 0 .
$$

Variables $s$ and $v$ are the real variables on the integration curve, measured as the curve length starting from the crack tip. With condition (2.8) equation (2.7) becomes a first kind Cauchy type singular integral equation and the integral is understood in terms of Cauchy principal value.

Following [2], the dislocation density function $b(s)$ in the form defined in (2.3-4), and consequently in the equation (2.10), does not correspond to a standard dislocation density used in fracture mechanics. The difference is in the coefficient. To use the standard definition, $d s$ has to be replaced by $d t$ in equations $(2.3,4)$ and $(2.10)$. This definition was used for the convenience of the numerical scheme only, and the difference is accounted for in the determination of the stress intensity factors. Essentially, the coefficient $d t / d s$ has been absorbed into the unknown dislocation density for computational convenience. However, 
the restriction on the integration path, following from the requirements of continuity of the derivative $d t / d s$, remains. Thus, the crack trajectory has to be a smooth curve with a continuous derivative.

Equation (2.10) is a homogeneous singular integral equation of the first kind on a semi-infinite interval; thus, the stabilization procedure has to be used in the formulation of the numerical procedure [18]. The regular numerical scheme is unstable because the solution of singular integral equations of this kind, generally, is not unique. The families of solutions of this equation are determined by the type of additional conditions imposed on the solution of the integral equation. One of these conditions can be an asymptotic behavior of the unknown function as $s \rightarrow \infty$. The behavior of $b(s)$ for large $s$ should correspond to remote loading conditions, or, as in the considered case, equivalently, to the reference loading. Thus, the dislocation density function can be written in the following form

$$
b(s)=K^{\infty}\left[\frac{\beta(s)}{\sqrt{s}}+i \frac{\left(1-e^{-s^{2}}\right)}{\alpha \sqrt{2 \pi s}}\right],
$$

where $K^{\infty}$ is remote (applied) stress intensity factor and function $\beta(s)$ is an unknown function bounded on the integration interval and

$$
\beta(\mathrm{s}) \rightarrow \mathrm{o}\left(\mathrm{s}^{-1 / 2}\right) \text { as } \mathrm{s} \rightarrow \infty
$$

The form chosen here is convenient for computations in the vicinity of the crack tip and the evaluation of the stress intensity factors at the crack tip, that is

$$
\frac{K_{I}^{0}+i K_{I I}^{0}}{K^{\infty}}=i \overline{\beta(0)} e^{i \theta^{0}}
$$

$\theta^{\circ}$ here is the angle of the tangential at the crack tip. $K$ with superscript o corresponds to local values of the stress intensity factors for Mode I and Mode II accordingly.

After substitution of (2.12) into (2.10) the equation is mapped onto a finite interval and 
then the collocation procedure is applied, in the form given by Rubinstein, [18], for the semi-infinite interval. This numerical procedure is based on the technique introduced by Erdogan and Gupta, [19].

The collocation scheme uses Gaus - Chebyshev quadrature formula and requires the node distribution along the roots of Chebyshev polynomials of the first and second kind. To secure the accuracy of the integration procedure, a nonlinear equation

$$
\int_{x^{0}}^{x_{k}}\left(1+\left(y^{\prime}(x)\right)^{2}\right)^{\frac{1}{2}} d x=s_{k}
$$

was solved to establish the relation between node $s_{k}$ and coordinates on the trajectory $x, y$. (Equation (2.13) corresponds to a mapped state.) $s_{k}$ here is a value of corresponding Chebyshev root, $x^{0}$ is a coordinate of the crack tip, and function $y(x)$ specifies the crack path.

\section{Observation of the experimental trajectories.}

The experimental crack path trajectories were analyzed by employing the technique described above. The experimental trajectories were approximated by polynomials using the list square method. These trajectories were used as initial data for the computational scheme. The computations were conducted for variable crack length along the given trajectories and for cases with a possibility of crack path deviations from the given experimental path.

The aim of the analysis is to establish a dominant characteristic parameter determining the crack path formation. In pursuit of this, the main fracture parameters are analyzed; namely, the focus is on the variations of the stress intensity factors and on the variation of the energy release rate due to the crack advance. The experimental specimen is illustrated in Figure 1a); the geometry of the active region is exaggerated there in order to make it visible on the figure. The maximal linear dimension of the region, where any significant interaction between the hole and a crack take place, is much smaller than the width of the specimen; therefore, small scale analysis can be applied. The resulting data are given as 
nondimensional values which were obtained by normalizing the stress intensity factors by the reference value of $K_{I}$ (illustrated in Figure 1b); the energy release rate is given as a value normalized by the corresponding reference value.

3.1 Variations of the crack tip parameters along the trajectories. The results of the computations for crack growth along the experimentally obtained trajectories are given in Figures 3-5. Cases marked on these figures correspond to trajectories marked on Figure 2. The data depicted in Figures 3-5 demonstrate that the crack growth is not characterized by monotonic increase of the stress intensity factor $K_{\mathrm{I}}$ or by zero value of $K_{\mathrm{II}}$ as is often expected. Trajectories 3 and 4 are at the distance of approximately $2 R$ ( $R$ is the radius of the hole), and cracks growing along these trajectories experience very weak interaction with the hole, although $K_{\text {II }}$ rises to about $5 \%$ of $K_{\mathrm{I}}$. Remarkably, cracks growing along the trajectories 1, 5, and 6 experience a very similar history for all analyzed parameters. Trajectory 2 is located in the region $R<y<2 R$ ( $y$ is a vertical coordinate of the trajectory origin with the center of the hole at $y=0$ ). This region is evidently characterized by a crack - hole interaction sufficient to turn the crack towards the hole, but not sufficient to maintain this direction, and eventually the crack returns to its original course.

The history of $K_{\mathrm{II}}$, Figure 4, is given in values normalized by the reference value of $K_{\mathrm{I}}$, which may be misleading in the way that the resulting data become relatively small. The values of $K_{\text {II }}$ vary in the range of $\pm 15 \%$ of the reference Mode I stress intensity factor. The generally accepted assumption that cracks grow in the direction of zero $K_{\mathrm{II}}$ is not supported by the obtained data. The possibility of numerical error was ruled out after multiple repetitions of the computations with different values of the parameters critical for the numerical scheme, such as number of nodes in the integration procedure, degree of the approximation polynomials, and so on. The frequency of the registered data points on the trajectories is sufficiently higher than the frequency of the oscillation of the values of $K_{\mathrm{II}}$, which rules out the possibility of insufficient mesh size in trajectory digitizing. Thus, it was made sure that the accuracy of the method of analysis is significantly higher than the 
observed amplitude of $K_{\text {II }}$ oscillation. It appears that any crack path trajectory with finite curvature will have some finite value of Mode II stress intensity component and it will most likely experience this kind of oscillation. The explanation of this will be given in the following section.

Relatively small values of the Mode II component of the local stresses make the energy release rate due to the crack advance behave similarly to the Mode I stress intensity factor. The $J$-integral is not path independent in the case of a curvilinear crack (this matter is discussed in [2]), and, additionally, due to the presence of the hole, the energy release of the

system cannot be associated with the local energy release at the crack tip. Thus, the traditional thought that the crack will propagate in the direction of maximal energy release of the system is not justifiable, and as is shown in Figure 5, the crack may propagate in the direction of decreasing energy release rate, as compared with its history along the crack path. This means that the decisive factor of the crack growth direction is a strictly local preference of the crack tip orientation.

The data obtained here cannot support the suggestion $[11,15]$ that the derivative $\partial K_{\mathrm{II}} / \partial L$ ( $L$ is the crack length) plays a significant role in the crack curving mechanism. However, one may make an argument against the importance of this derivative, since, as the data show below, the local variations with respect to a potential deflection angle are more important for the crack path formation, than the history type dependence on the crack length increment.

\subsection{Variations of the crack tip parameters with respect to possible crack path deflections.}

In this part of the investigation the crack propagation of the numerical model was assumed to be partially along the given trajectory up to certain point, and then small perturbations of the experimental path were given. These perturbations were given in the form of a circular arc tangential to the original path and small in length if compared with any geometrical parameter of the problem. The length of these arcs was chosen so small that it did not affect the result, and the only important parameter that remained was the angle formed by the 
tangential at the free end of the arc and by the tangential to the actual crack path trajectory at the point correspondent to the increment of the crack advance equal to the arc length. These crack path perturbations are illustrated schematically in Figure 6. The length of the arcs is enlarged in Figure 6 for better visualization. The sign of the angle of the crack path deflection is marked in Figure 6.

The aim of this analysis is to determine what makes the actual crack path preferable with respect to other possible crack path directions. The results are given in Figure $7 a-d$ for three crack tip positions $x_{\circ}=2.0,3.0,4.0$ and deflections of \pm 10 degrees. The variation of the Mode II stress intensity factor is given for one position only because it exhibits practically equivalent behavior for all cases considered. Variations of the Mode I stress intensity factor are given for all three cases. The data for the energy release rate are omitted here since they practically duplicate the variations of $K_{\mathrm{I}}$ with changed scale.

The data for $K_{\mathrm{I}}$ are normalized by the value of $K_{\mathrm{I}}(\theta=0)$ corresponding to zero deflection angle, and the values of $K_{\text {II }}$ are normalized by the value of the reference Mode I stress intensity factor, as in the previous section.

None of the considered positions can be described as zero $K_{\text {II }}$ direction, as a direction of maximal $K_{\mathrm{I}}$, or as a direction of maximal energy release rate due to the crack advance. By the directions of maximal value here, one means maximal value with respect to an angle of possible crack path perturbations. Directions of maximal energy release rate due to the crack advance are slightly off the course of the crack path. These directions practically coincide with $K_{\mathrm{II}}=0$ direction and, therefore, coincide with maximal $K_{\mathrm{I}}$ direction, as it was predicted by Cotterell and Rice [9]. On Figure $7 b$, directions of zero $K_{\mathrm{II}}$ are marked on the curves. The difference between the maximal $K_{\mathrm{I}}$ directions and the marked directions of $K_{\mathrm{II}}=0$ is less than 0.2 degrees, and this is a well reproduced result. The difference between the actual crack path direction and direction of a maximal energy release varies between 2 to 15 degrees. Denoting the deflection angle $\alpha$ and the energy release rate per crack advance $G$, we observe that for higher value of the derivative $\partial G / \partial \alpha$ at $\alpha=0$, the direction 
of the maximal $G$ is close to the actual path. It seems to be more suitable physically to describe this process in terms of the energy release rate rather than in terms of stress intensity factor, although quantitatively both would give the same result, since direction of maximal $K_{\mathrm{I}}$ coincides with direction of zero $K_{\mathrm{II}}$.

\section{Proposed crack path deflection mechanism.}

To explain the observed data which were described in the previous section, it is essential to consider the material properties exhibited during the crack growth process. As was pointed out in the introduction, the material which was used in the experiment may be characterized by the formation of a narrow nonlinear zone along the crack path. In the framework of linear fracture mechanics, the crack and the surrounding zone cannot be separated and are treated as a single object. The resulting fracture mechanics parameters then may be interpreted as remote, or applied, values controlling the nonlinear region. The nonlinear zone acts as a buffer for the crack in absorbing the asymmetry of the applied stress field. As a result, the nonlinear zone becomes asymmetric with respect to a crack line. The nature of the nonlinear deformation mechanism is not important in principle, and for purposes of this study, it may be characterized by the quantity of the stress field asymmetry it can absorb before turning the crack. In the framework of linear fracture mechanics, the derivative $\partial G / \partial \alpha$ at $\alpha=0$ and $K_{I I}$ are the only parameters that may be used as a measure of the asymmetry of the applied stress field acting in the vicinity of a crack tip. Of these two parameters, the energy release gradient with respect to the deflection angle, $\partial G / \partial \alpha$, has a better physical interpretation in terms of the energy release rate increase (benefit) due to the crack tip rotation. It is a material property that determines the critical value of the energy release rate benefit required to rotate the crack tip. On the other hand, considering that both parameters play similar roles, one has to note that the critical value of $K_{\mathrm{II}}$ would be easier to measure. In Figure 8, schematic development of an asymmetric crack propagation process zone is illustrated. Thus, 


$$
S=\frac{a-b}{a+b} \propto \frac{\partial G}{\partial \alpha} \quad(\alpha=0)
$$

where $b$ and $a$ are linear dimensions of the wake of the nonlinear process zone, as illustrated in Figure 8. The ratio $S$ on the right of (3.1) may be taken as a geometric criterion of the asymmetric development of the nonlinear region. For positive values of $a$ and $b$, this ratio changes from -1 to 1 , with 0 corresponding to the symmetric case. Sign of $S$ corresponds to the direction of the angle of deflection.

The critical value of the energy release rate gradient at which material will require a change in crack growth direction may determine the instant at which the crack tip orientation will change, but it cannot, generally speaking, determine the amount of crack tip deflection because of the buffering effect of the nonlinear process zone. The new crack growth direction will most likely be dictated by the microstructure of the material at the particular instant, such as the grain size, or local orientation of crystallographic planes. At this point, the crack will continue to grow in that direction until the critical situation develops again, and the energy release rate gradient reaches the critical value for the material; consequently, the crack growth direction will change again. Thus, the ductile materials with high buffering capability can tolerate a higher degree of stress field asymmetry, and require smaller crack path alterations to accommodate it. Therefore, ductile materials will exhibit smooth crack path trajectories, and brittle materials with a very narrow nonlinear zone or without this buffering zone will exhibit sharp turns along the crack growing path. This type of observation was reported in [20].

In the analyzed experiment, the critical value of the energy release rate gradient (or critical value of $K_{\text {II }}$ ) was never reached in case 4 , and it was nearly reached in case 3 . In other cases, as the curvature of the trajectory changes, the crack path experiences a sequence of corrections in its direction; this explains the oscillation of $K_{\text {II }}$ observed earlier. The frequency of this oscillation is determined by the material property associated with a critical energy release rate gradient and by the intensity of the applied stress field. Case 2 clearly demonstrates this oscillation of $K_{\mathrm{II}}$, as is shown in Figure 4. 
It follows, that under mixed mode loading, only the ideally brittle materials are capable of forming a crack path with $K_{\mathrm{II}}=0$ along the significant crack path segments, and these segments will be nearly straight line intervals.

\section{Discussion and conclusions.}

The experimental crack path trajectories were analyzed to establish the governing mechanism of the crack path formation. The experimental data were supplied by the authors of reference [1]. The experimental crack path trajectories were used as boundary value data for the numerical analysis. Through accurate numerical procedure the essential fracture mechanics parameters were analyzed as they develop along the practical crack path trajectories and as they change due to crack path perturbations.

Results reported here were reproduced from data from different specimens, which shows their reliability.

It was observed that the crack path is not characterized by monotonic increase of the energy release rate due to the crack advance, and the crack growth direction is controlled by local gradients of the energy release rate with respect to a deflection angle.

The proposed crack path formation mechanism is based on the assumption that the process zone formed during the crack growth serves as a buffer between the applied load asymmetry and the crack tip reaction to it. Thus, the magnitude of the energy release rate gradient, required to alter the crack growth direction, is associated with the process zone in the vicinity of the crack tip. The ability of this zone to transmit the asymmetric properties of the applied stress field is a material property, and is important for the crack path alteration. In other words, the buffering properties of the process zone must be characterized by the material constant. This constant can be associated with the maximal value of the energy release rate gradient with respect to deflection angle, which the crack process zone can tolerate without a change in crack growth direction. This property of the material may be described as a maximal allowable geometrical asymmetry of the process zone, which can be specified by the parameter $S$ introduced in equation (3.1). 
The results of the numerical analysis show that the direction of the maximal energy release rate due to the crack advance coincides with the direction of the zero Mode II stress intensity factor, which is consistent with [9]. Therefore, the critical value of $K_{\text {II }}$, which determines the instant when the crack tip changes orientation for a particular material, can be used as an equivalent to the energy release rate gradient. The disadvantage of using the $K_{\text {II }}$ criterion is that for the crack growth, the certain value of the Mode I stress intensity factor has to be maintained, and the combination of both Modes of the applied stress field is important.

As the crack tip approaches the inhomogeneity, the magnitude of the energy release rate gradient increases; after it reaches the critical value for the material, the crack growing direction changes until the critical value of the gradient is reached again. If the material is homogeneous, this process can be accomplished in small crack growth increments forming a smooth crack path curve. As was mentioned, the Mode II stress intensity factor plays a role similar to the energy release rate gradient in controlling the crack path. The curvilinear crack path experiences continuous correction, and, therefore, the oscillations of $K_{\text {II }}$ are observed along the crack path. Accordingly, only in very brittle materials with negligible nonlinear process zone size, the crack path may exhibit sharp turns and may have straight line intervals of significant length with near zero values of $K_{\mathrm{II}}$.

\section{Acknowledgment}

This work was supported by NASA Lewis Research Center under Grant NAG 3-815 and by the Institute for Computational Mechanics in Propulsion at NASA Lewis Research Center. The author would like to thank Professor Chudnovsky for several useful discussions and for sharing experimental data in greater detail than appeared in publication [1].

\section{References}

1. A. Chudnovsky, K. Chaoui, A. Moet, "Curvilinear Crack Layer Propagation." Journal of Material Science Letters Vol. 6 (1987) pp. 1033-1038

2. A. A. Rubinstein, "Crack Path Effect on Material Toughness." Journal of Applied 
Mechanics. Transactions of the ASME. (in press)

3. Erdogan F., Sih G. C., "On the Crack Extension in Plates under Plane Loading and Transverse Shear." Journal of Basic Engineering, Transactions of the ASME, 1963, pp. $519-525$.

4. Goldstein, R. V., "Plane Problem of a Curvilinear Cracks in an Elastic Solids." Mechanics of Solids, Izv. ANSSR, 1970, pp. 69-82.

5. Banichuk, N. V., "Determination of the Shape of a Curvilinear Crack by Small Parameter Technique." Mechanics of Solids, Izv. ANSSR, 1970, pp.131-137.

6. Smith, D. G. and Smith, C. W., "Photoelastic Determination of Mixed Mode Stress Intensity Factors." Engineering Fracture Mechanics. Vol. 4, pp. 357-366, 1972.

7. Wu, C. H., "Fracture under Combined Loads by Maximum-Energy-Release-Rate Criterion." Journal of Applied Mechanics, vol. 45, pp. 553-338, 1978.

8. Wu, C. H., "Explicit Asymptotic Solution for the Maximum-Energy-Release-Rate Problem." Int. J. Solids Structures, vol. 15, PP. 561-566, 1979.

9. Cotterell, B. and Rice, J. R. "Slightly curved or kinked cracks." Int. J. Fracture, Vol. 16, No. 2, pp. 155-169, 1980.

10. Karihaloo, B. L., Keer L. M., Nemat-Naseer, S. N., "Crack Kinking Under Nonsymmetric Loading." Engineering Fracture Mechanics, vol. 13, pp. 879-888, 1980.

11. Karihaloo, B. L., Keer, L. M., Nemat-Nasser, S., Oranratnachai A., "Approximate Description of Crack Kinking and Curving." Journal of Applied Mechanics, 1981, vol. 48, PP. 515-519, 1981.

12. Sheng, C. F., Wheeler, L., "Crack Path Prediction for a Kinked Crack in the Neighborhood of a Circular Inclusion in an Infinite Medium." Journal of Applied Mechanics, vol. 48, 313-319, 1981.

13. Hayashi, K., Nemat-Nasser, S., "Energy-Release Rate and Crack Kinking under Combined Loading." Journal of applied Mechanics, vol. 48, pp. 520-524, 1981.

14. Karihaloo, B. L., Nemat-Naser, S., "Thermally Induced Crack Curving in Brittle Solids." 
Proceedings of the International Conference on Analytical and Experimental Fracture Mechanics, (G. C. Sih and M. Mirabil ed.) Noordhoff, 1981, pp. 265-272.

15. Karihaloo, B. L., "On Crack Kinking and Curving." Mechanics of Materials, Vol. 1, pp. 189-201, 1982.

16. Sumi, Y., Ohashi, K., Emura, H., "On Crack Arrestability by a Circular Hole Based on Computational Crack Path Prediction." Dept. of Naval Architecture and Ocean Eng., Yokohama National University.

17. Muskhelishvili, N. I. Some Basic Problems in the Mathematical Theory of Elasticity. P. Noordhoff, Ltd., Holland, 1963.

18. Rubinstein, A. A., "Macrocrack - Microdefect Interaction." ASME Journal of Applied Mechanics, Vol. 53 pp. 505 - 510, 1986.

19. Erdogan, F. and Gupta, G. D. "On the Numerical Solutions of Singular Integral Equations." Quarterly of Applied Mathematics, Vol. 29, pp. 525 - 534, 1972.

20. Bazzard, R. J., Gross, B. and Srawley, J. E. "Mode II Fatigue Crack Growth Specimen Development." ASTM, STP 905, pp. 329-346, 1986.

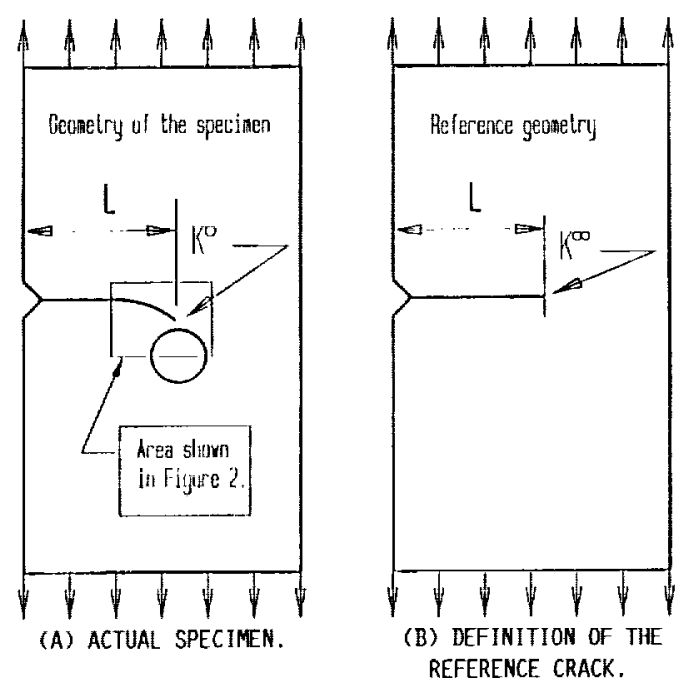

FIGURE 1. - GEOMETRY OF THE SPECIMEN.

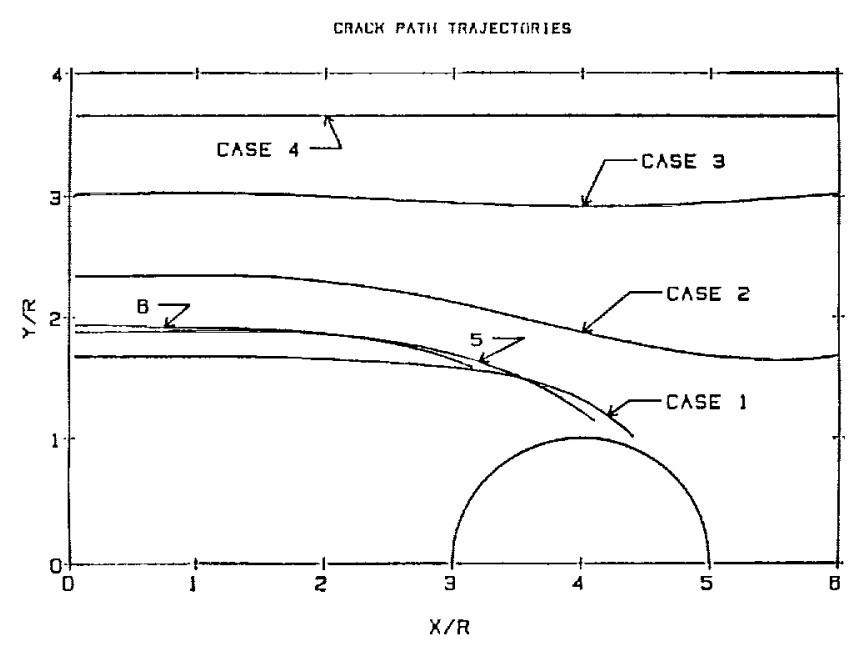

FIGURE 2. - EXPERIMENTAL CRACK PATH TRAJECTORIES (DATA FOR THESE TRAJECTORIES WERE SUPPL IED BY AUTHORS (1]). 
KI OF LUROYINE CRACKg

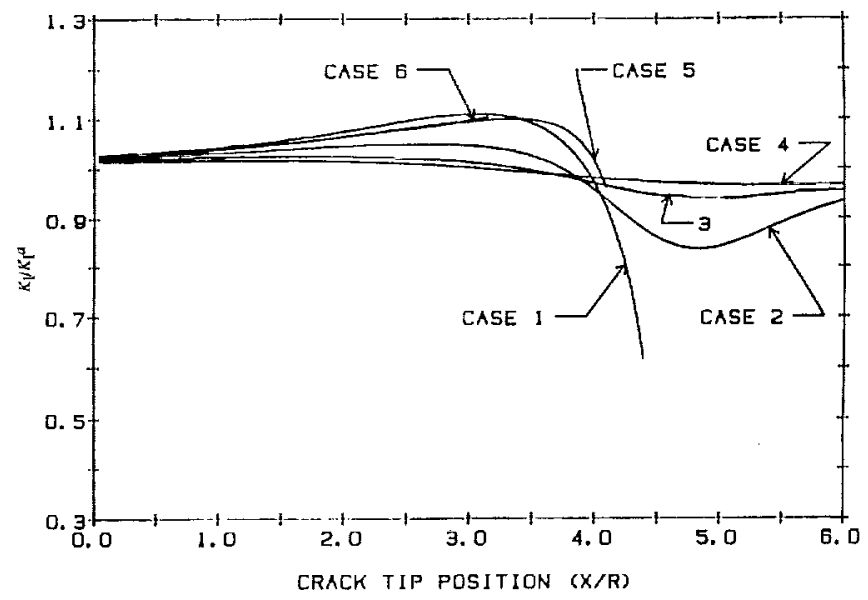

FIGURE 3. - K VARIATION ALONG THE CURVILINEAR CRACK PATH TRAJECTORIES.



FIGURE 4. - K K VARIATION ALONG THE CURVILINEAR CRACK PATH TRAJECTORIES.
TRAJECTORY ANALYYIS

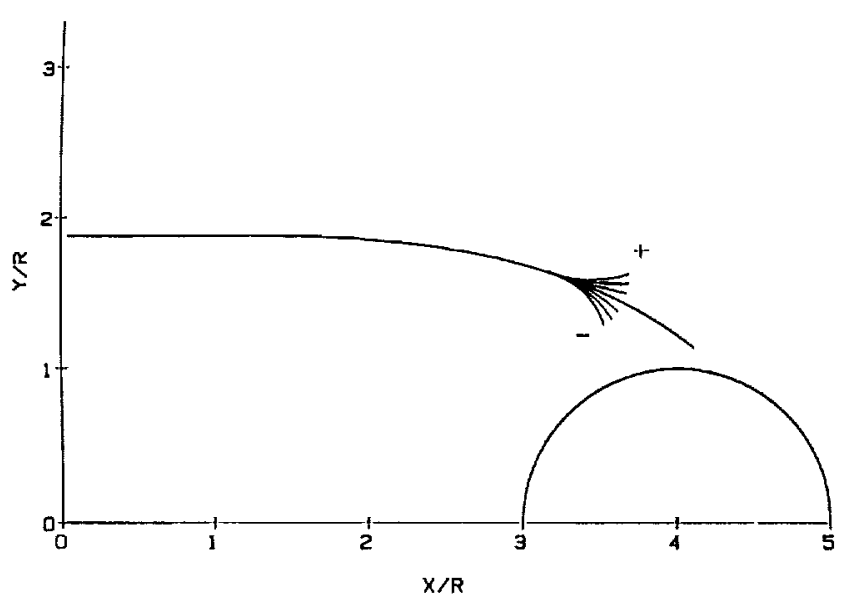

FIGURE 6. - CRACK PATH DEFLECTIONS IN THE FORM OF CIRCULAR ARCS USED IN THE ANALYSIS OF THE LOCAL VARIATIONS OF THE FRACTURE MECHANICS PARAMETERS. THE LENGTH OF THE ARCS IS EXAGGERATED IN THIS ILLUSTRATION. 
K1 V5. AMFLE AT CT-2. DO



(A) CRACK TIPS AT $X / R=2$.

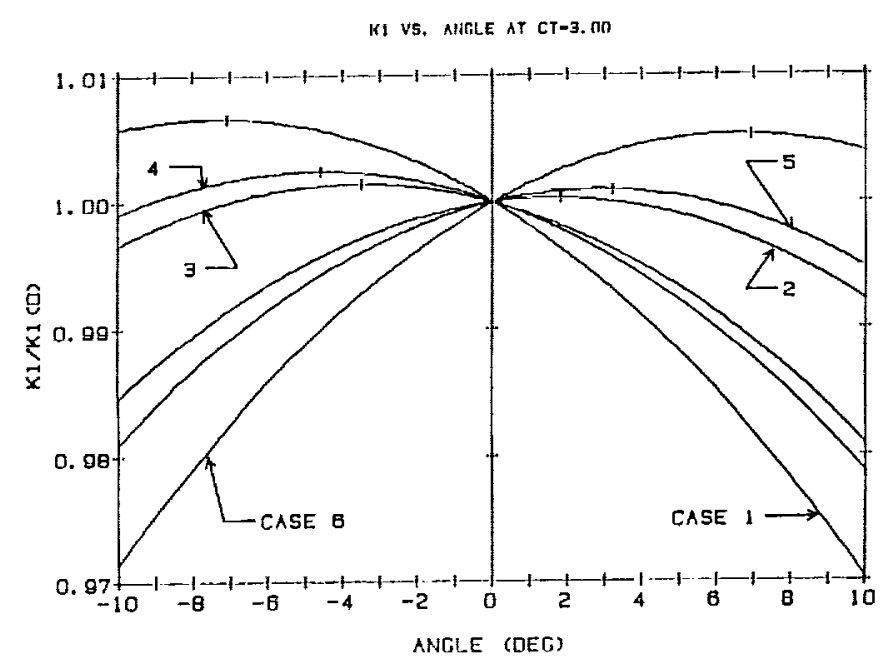

(B) $x / R=3$.
HI VS. AIGLLE AT CT-4. OOI

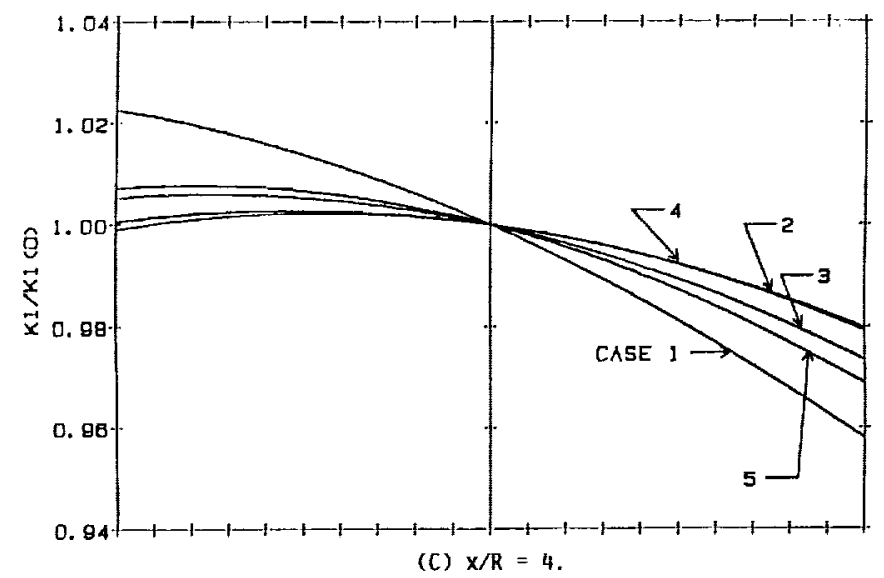

K2 vS. AHGLE AT CT-3. ON

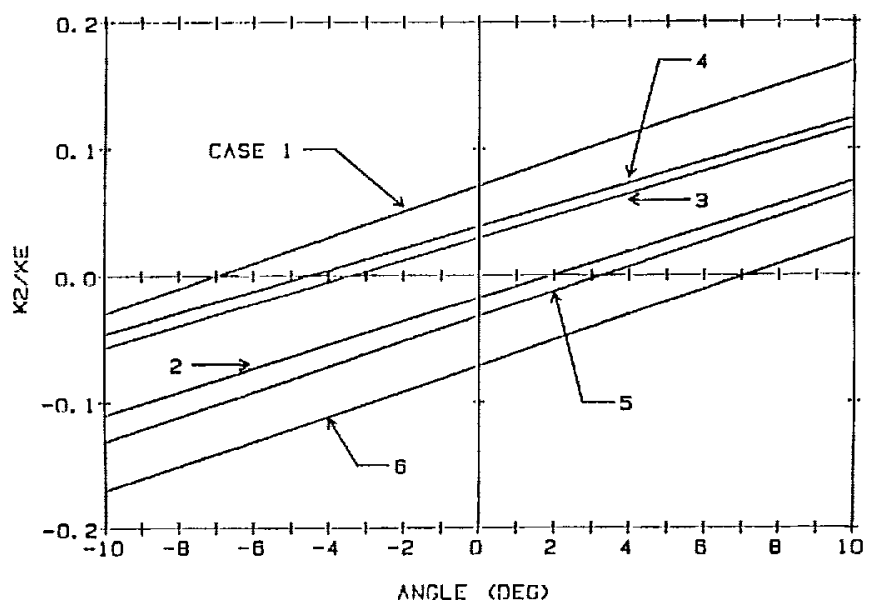

(D) VARIATIONS OF $K_{I I}$, CRACK TIPS AT $x / R=3$.

FIGURE 7. - LOCAL VARIATIONS OF K I DUE TO CRACK PATH REFLECTIONS. THE CENTER OF THE HOLE IS AT $x / R=4$.

\section{1}

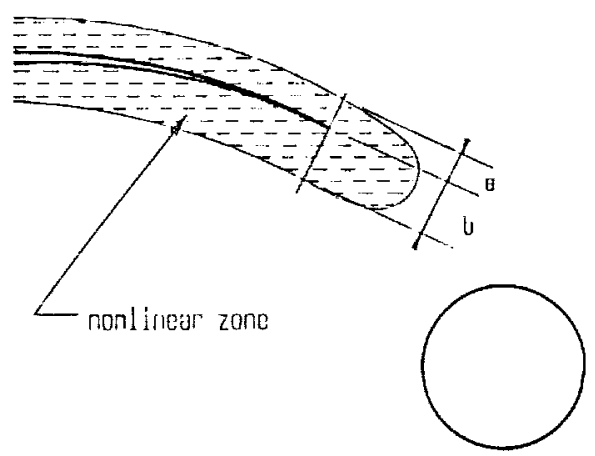

\section{$1+1+1+1+1+1+1+1+1+1$}

FIGURE 8. - NONLINFAR ZOME SIIRROINDING THE CURVILINEAR CRACK. 


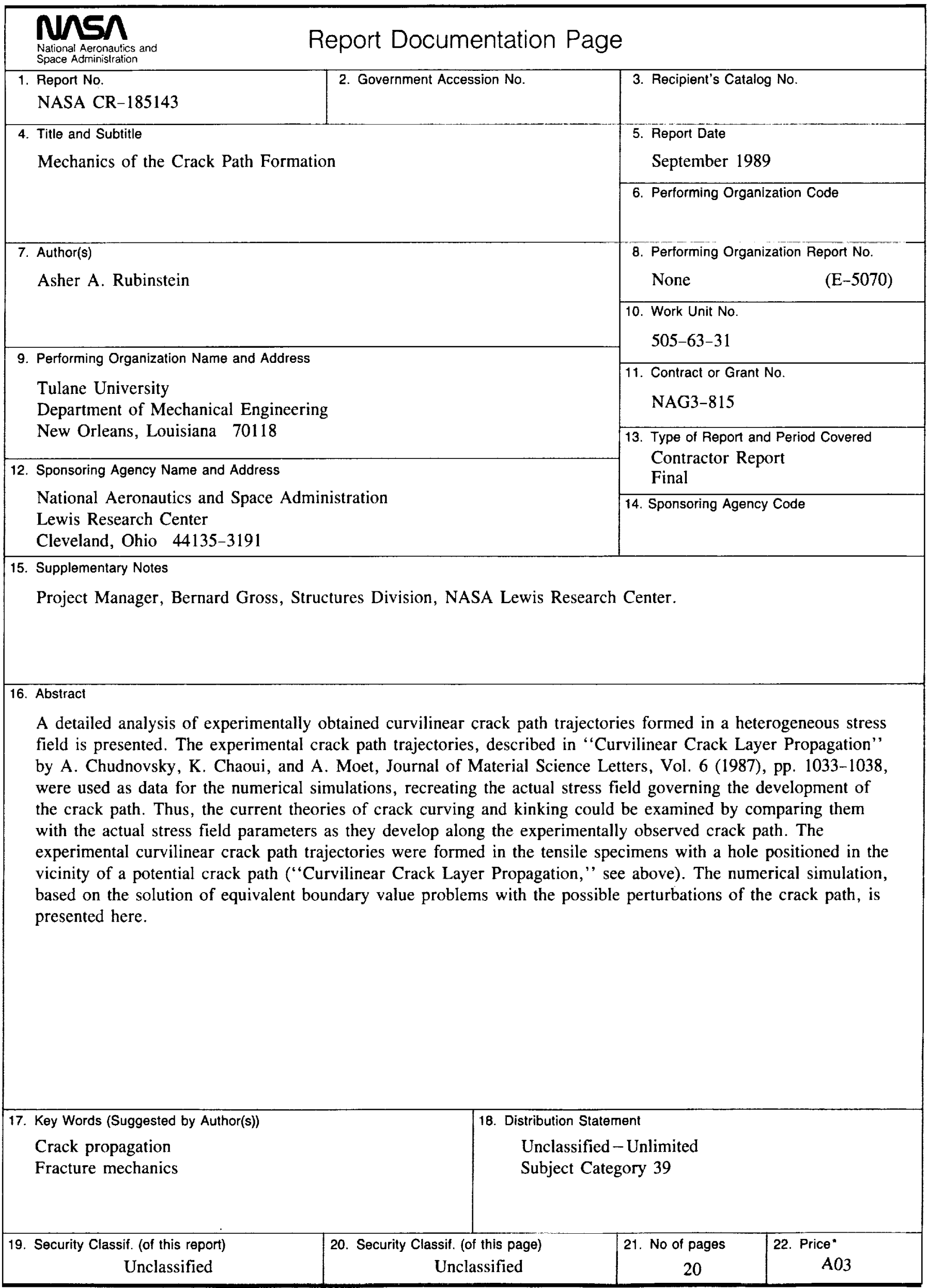


